\title{
Experimental data for groundwave propagation over cylindrical surfaces
}

King, Ray J.; Cho, Se.; Jaggard, D.; Brackner, G.; Hustig, C.

Published in:

I E E E Transactions on Antennas and Propagation

Publication date:

1974

Document Version

Publisher's PDF, also known as Version of record

Link back to DTU Orbit

Citation (APA):

King, R. J., Cho, S., Jaggard, D., Brackner, G., \& Hustig, C. (1974). Experimental data for groundwave propagation over cylindrical surfaces. I E E E Transactions on Antennas and Propagation, 22(4), 551-556.

\section{General rights}

Copyright and moral rights for the publications made accessible in the public portal are retained by the authors and/or other copyright owners and it is a condition of accessing publications that users recognise and abide by the legal requirements associated with these rights.

- Users may download and print one copy of any publication from the public portal for the purpose of private study or research.

- You may not further distribute the material or use it for any profit-making activity or commercial gain

- You may freely distribute the URL identifying the publication in the public portal

If you believe that this document breaches copyright please contact us providing details, and we will remove access to the work immediately and investigate your claim. 


\title{
Experimental Data for Groundwave Propagation over Cylindrical Surfaces
}

\author{
RAY J. KING, MEMBER, IEeE, SE H. CHO, MeMber, IEeE, DWIGHT L. JAGGARD, sttdent MEMrber, IEee, \\ GARRETH E. BRUCKNER, MEMBER, IEEE, AND C. H. HUSTIG
}

\begin{abstract}
Experimental data for the fields of EM groundwaves propagating over cylindrical homogeneous paths and two-section mixed paths were obtained by microwave $(4.765 \mathrm{GHz})$ modeling. The cylindrical surfaces, which have a radius of $20 \lambda_{0}$, closely approximate spherical surfaces insofar as groundwave propagation is concerned. The model is a curved tank which was constructed as a stratified combination of Plexiglas over distilled water, giving a predictable highly inductive surface impedance. Aluminum foil laid on the Plexiglas produced a nearly perfectly conducting surface wherever needed for the mixed path cases. For both homogeneous and mixed paths the residue series theory agrees well with the experimental data in the shadow and penumbra regions, but the agreement becomes poorer as the observation point moves into the illuminated region where an excess number of residue terms is needed. The results show that a groundwave which crosses an abrupt boundary rapidly acquires the characteristic properties of the second medium at small heights above the surface, and that the equivalent integral solution for mixed paths is therefore adequate close to the surface. This is especially useful near the boundary where the residue series converges poorly. It is concluded that if the constitutive electrical parameters of the earth are precisely known and constant, the theory can be reliably applied to LF and VLF groundwave propagation over the earth where the constraints are even less severe.
\end{abstract}

\section{INTRODUCTION}

$\mathbf{T}$ THE THEORY for groundwave propagation over smooth spherical homogeneous and mixed paths has been developed theoretically and numerically using various methods, and there is little doubt about the correctness of the theory. However, reliable experimental data does not exist for either case, and so the experiments reported here were designed to confirm the theories developed from the corresponding idealized mathematical

Manuscript received April 23, 1973: revised February 14, 1974. This work was supported in part by the Tational Science Foundation under Research Grants GK-2311 and GK-21218, and in part by the University of Wisconsin (rraduate School Research Committee. This paper was presented at the Fall LSNC/LRSI Meeting, Williamsburg, Ya., 1)ecember $12-15,1972$.

IR. J. King is with the Laboratory for Electromagnetic Theory, Technical Cniversity of Demmark, Lyngby, Denmark, on leave from the Department of Electrical and Computer Engineering, Cniversity of Wisconsin, Madison, Wis. 53706.

s. H. Cho is with the Department of Electrical and Computer Engineering, Univer ity of Wisconsin, Madison, Wis. 53706.

I). I. Jaggard was with the I epartment of Flectrical and Computer Engineering, University of Wisconsin, Madison, Wis. He is now with the Jet Propulsion Laboratory, Californis Institute of Technology, Pasadena, Calif.

G. E. Bruckner was with the Iepartment of Electrical and Computer Engineering, Lniversity of Wisconsin, Madison, Wis. He is now with the Bechtel Corporation, San Francisco, Calif.

C. H. Hustic was with the Iepartment of Electrical and Computer Engineering, L'niversity of Wisconsin, Madison, Wis. 53706. models. When this is done other work can follow which accounts for such factors as the ionosphere, the earth's anisotropy, nonuniformity, roughness and the presence of hills, cliffs, sloping beaches, ete.

But these factors make the cost prohibitive and it is nearly impossible to gather highly accurate experimental data from a large scale situation using, say, Loran C transmissions over the real earth. The problems are compounded by weather and season-to-season variations. Data along the earth's surface would have to be gathered using mobile land vehicles, ships or planes, while heightgain experiments would require rockets or balloons, all of which have problems of their own.

Well simulated experiments which use microwave models overcome all of these problems sinee the parameters and variables can be carefully chosen and controlled in the laboratory. But, as we shall see, modeling also has problems of its own.

It should be noted at the very outset that the "attenuation function" for propagation over a cylindrical surface presumably differs negligibly from that of a sphere for grazing propagation along the circumference of the surface when the radius $a$ is several free space wavelengths [1], [?].

The experiments described here used microwave frequency $\left(4.76 \tilde{\mathrm{j}} \mathrm{GHz}, \lambda_{0}=6.3 \mathrm{~cm}\right)$ crlindrical models. The radial electric field over the surface was measured using a small scattering dipole and a homodyne detoction system. This technique has proven to be highly sensitive and accurate in previous modeling experiments $[3]-[7]$.

The goal of this work is to determine the accuracy of the theory for various variables such as receiver location. along the great circle, receiver height in the shadow, penumbra or illuminated regions, or its location near an abrupt boundary separating two media. Materials were therefore chosen which give a large contrast of surface impedances for the mixed paths to enhance scattering and diffraction from the boundary while the cylinder radius $\left(\cong 20 \lambda_{0}\right)$ was chosen to enhance the effect of diffraction by surface eurvature.

\section{THE EXPERILENT}

The model experiments were performed on a cylindrical water tank design approximately $0.9 \mathrm{~m}$ wide, having an are length of $1.12 \mathrm{~m}$ and a radius of $a=1.25 \mathrm{~m}\left(\simeq 20 \lambda_{0}\right)$. The TMI wave source was an open-ended waveguide 


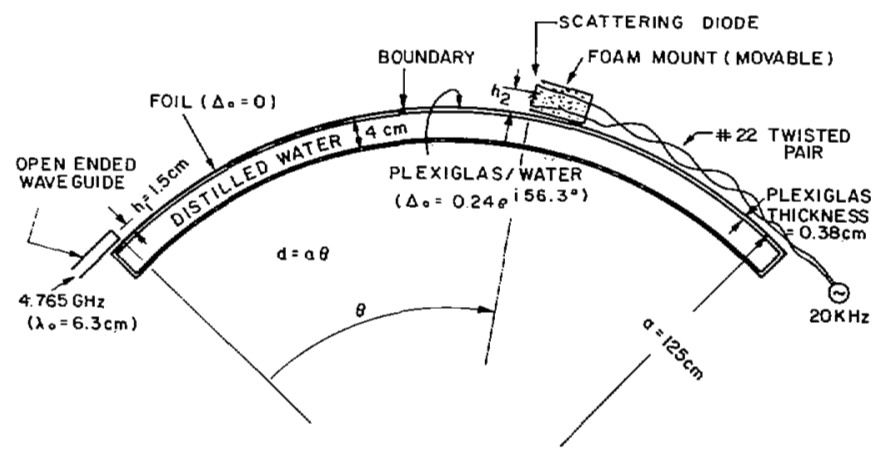

Fig. 1. Cross section of cylindrical Plexiglas water tank. Openended waveguide was used as transmitting antenna and modulated scattering diode (dipole) was used as probe to measure radial electric field.

( $C$ band) having the plane of its aperture normal to the surface, centered at one end of the surface, and radiating approximately $250 \mathrm{~mW}$ along the curved $1.12-\mathrm{m}$ dimension. As shown in Fig. 1, the water tank model was constructed of Plexiglas, the top cover being $0.302 \mathrm{~cm}$ thick, and the distilled water depth being $4 \mathrm{~cm}$. This depth is sufficient to provide a surface impedance which is essentially the same as that for an infinite water depth. This was checked by assuming a metal bottom (worst case) and computing the change in the surface impedance as a function of water depth. Similarly, variations of the surface impedance due to the thickness tolerance of the top cover $( \pm 0.03 \mathrm{~cm})$ were found to be insignificant. From a precise knowledge of the constitutive electrical parameters of both media $\left(\mu_{0}, \epsilon, \sigma\right)$, the surface impedance normalized by $\eta_{0}(=120 \pi)$ for this stratified combination of Plexiglas and water was calculated to be $\Delta=Z / \eta_{0}=$ $0.24 e^{i 56.3^{\circ}}$. A second surface impedance of $\Delta=0$ could easily be obtained anywhere on the cylindrical surface by placing 0.0125 -cm thick aluminum foil with an adhesive backing over the Plexiglas cover.

This particular combination of materials was chosen because the surface impedances were accurately known and give a large contrast between the two surfaces, making the observed effects large when propagating over mixed paths. Furthermore, the Plexiglas and water medium gives a highly inductive surface on which a trapped surface wave can propagate, in addition to the usual surface wave which exists for smaller arguments of $\Delta[8]$.

For this model, the real earth scaled frequency is approximately $1 \mathrm{kHz}$ (for $a / \lambda_{0} \cong 20$ ), and so the theory will be more accurate when applied to large scale groundwave propagation over the earth in the VLF and LF ranges, e.g., $a / \lambda_{0} \cong 64$ at the low end of the VLF band $(3 \mathrm{kHz})$. This is approaching the lowest frequency where this groundwave theory can be applied, since the effects of the ionosphere must be included at lower frequencies, especially at ranges beyond a few hundred kilometers.

The radial electric field $E_{r}$ versus $d$ and $h_{2}$ was measured using a short $\left(0.1 \lambda_{0}\right)$ radially oriented scattering diode which was switched by a $20 \mathrm{kHz}$ generator through a B\&S No. 22 twisted pair transmission line as shown in
Fig. 1. The open-ended waveguide served as both the CW power transmitting antenna and the receiving antenna for the modulated back-scattered wave, while a hybrid tee arrangement (not shown) separated the backscattered signal from the outgoing $C W$ wave. This backscattered signal is proportional to $E_{r}^{2}$ and was detected with an error less than $0.2 \mathrm{~dB}$ by a previously developed homodyne detection system [5].

In all measurements the twisted pair transmission line was oriented normal to the radial electric field, and brass loading disks (diameter $=0.65 \mathrm{~cm}$, unless otherwise noted) were placed on the ends of the scattering diode leads to increase the scattering efficiency. Unfortunately, at very low heights these loading disks and also the twisted pair line enhance the electrostatic coupling of the scattering diode (dipole field probe) with the surface, thereby altering the $\mathrm{RF}$ impedance of the dipole. This in turn altered the scattered field. The difficulty with the leads was essentially eliminated by inserting a $20-\mathrm{cm}$ section of special plastic coated, high RF loss semiconducting leads between the diode and the twisted pair line.

The modeling and field probing techniques used here are essentially the same as those previously used for measuring the electric field over a flat surface, and the reader is referred to King et al. [6] for more details

\section{COMIPARISON OF THEORY AND EXPERIMIENTAL RESULTS}

Excellent reviews of the theory and the most well known formulas have been given by Wait $[1],[9],[11],[12]$ and Logan and Yee [10], and so the reader is referred to these for more details and other references All equations in the following discussion can be found in Wait [1], and the equation or page numbers will be noted as they are needed. The assumed excitation is $\exp (i \omega t)$.

In the following theoretical calculations, the transmitting antenna height was taken to be $1.5 \mathrm{~cm}$ which is slightly higher than the geometrical center of the openended waveguide. This height cannot be estimated theoretically and so it was necessary to determine its value by comparing the theory with experimental data for several different values of $h_{2}$.

For the convenient presentation of data, several curves for different parameter values appear on the same figure, but it is to be understood that these curves are all relative to each other.

The radial electric field at $(r, \theta)$ for a Hertzian electric dipole with current moment $I d l$, which is radially directed and located in free space at $(b, 0)$ over a sphere of radius $a$, can be written

$$
E_{r}=\frac{I d l k \eta_{0}}{2 \pi i} \frac{a \exp (-i k d)}{b r(\theta \sin \theta)^{1 / 2}} W^{\prime}\left(d, Z_{1}, Z_{2}\right)
$$

where $k$ is the free-space wavenumber, and $d(=a \theta)$ is the great circle distance along the surface of the sphere. The boundary between the two media, each having surface impedance $Z_{1}$ and $Z_{2}$, lies perpendicular to the propagation 
path at $d=d_{1}$. Note that as $r$ and $b$ approach $a$ and in turn $a \rightarrow \infty$, the coefficient of $W^{\prime}$ is the electric field over a perfectly conducting plane. Thus $W^{\prime}\left(d, Z_{1} Z_{2}\right)$ is the spherical mixed path attenuation function which accounts for the effect of the two surface impedances $Z_{1}$ and $Z_{2}$, and in part, for the diffraction of the curved surface.

The height-gain factor for the receiver is defined as the ratio of the total field at height $h_{2}(=r-a)$ to that for $h_{2}=0$

$$
G\left(h_{2}\right)=\frac{E_{r}\left(d, h_{1}, h_{2}\right)}{E_{r}\left(d, h_{1}, 0\right)}
$$

where $h_{1}(=b-a)$ is the source height.

\section{A. Homogeneous Paths}

When the receiver is located in front of the boundary, i.e., $d<d_{1}$, the field is essentially that for a homogeneous path of impedance $Z_{1}$. Of course, this neglects the wave reflected from the boundary, but this wave has been shown to be quite small on a number of occasions, both analytically [e.g., see $[11],[12],[14],[15]$ and experimentally [3], [4], [7] using planar models similar to those described here. Then, for a homogeneous surface or when $d<d_{1}$, $W^{\prime}\left(d, Z_{1}, Z_{2}\right) \cong W\left(d, Z_{1}\right)$ which is given in the form of a residue scries in $[1,(131)],[11,(95)],[12,(3)]$. This representation is valid for $\left(h_{1}+h_{2}\right) \ll a,|\Delta|^{2} \ll 1$ and large spheres where $k a \gg 1$.

A very close correspondence exists between the residue series solution for a vertical electric dipole exciting a sphere [1]. [9], [12] and a line source exciting a cylinder [11]. As it happens, the resulting approximated solutions for $W$ in these two cases are identical, the only differences being in the spreading factor and the focusing effect at the antipode. The antipodal focus for a sphere is an axial caustic, and a line focus for the cylinder. How well this correspondence carries over to the case of the dipole excited cylinder being experimentally considered here is an open question which should be studied further. But we suggest, and Wait [13] concurs, that such an equivalence is likely to only exist for $d<a$, in view of the difference in transverse curvature for the sphere and the cylinder. Put another way, the transverse curvature should not differ significantly over the first few Fresnel zones of maximum width $\left(m \lambda_{0} d / 2\right)^{1 / 2}, m=1,2, \cdots$, if both sources are dipoles. Such a restriction is likely to be particularly important in mixed path propagation where significant forward scattering from those portions of a boundary lying to the sides of the great circle path ean occur $[1$, p. 204], [12].

Since the only difference bet ween the cases of the sphere with a dipole source and the cylinder with a line source is the spreading coefficient, the simplest of both cases was used in these experiments, e.g., a dipole (whose vertical radiation pattern is closely approximated by an openended waveguide) above a cylindrical model (which is much easier to construct). This model also gives some experimental check on the equivalence of the field for a

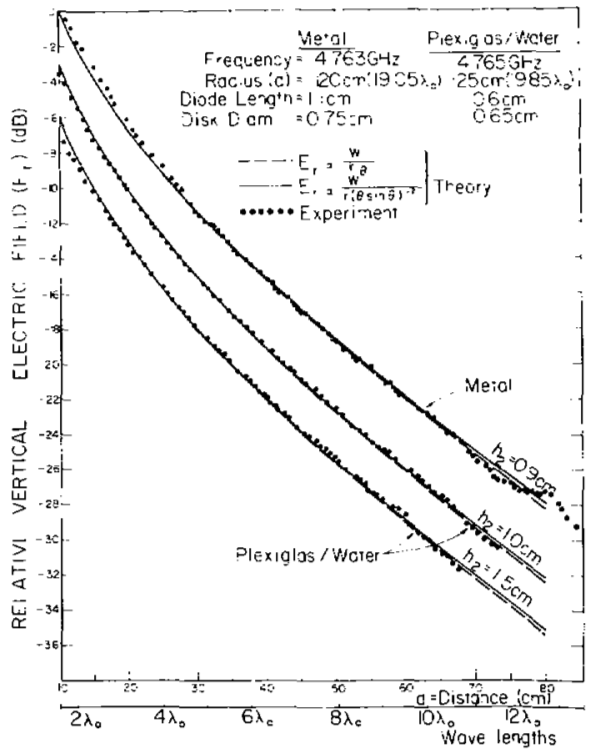

Fig. 2. Dipole excited groundwave field versus distance $d \quad(=a \theta)$ over homogeneous cylindrical (experimental) and spherical (theory) surfaces of metal $(\Delta=0)$ and Plexigla: over water $\left.\Delta=0.24 e^{i 36.30}\right) \cdot h_{1}=1.5 \mathrm{~cm}$.

dipole excited cylinder and the theory for the dipole excited sphere.

Csing $W\left(d, Z_{1}\right)$ in (1), the solid curve in Fig. 2 shows the calculated radial electric field versus distance for metal and Plexiglas and water homngeneous paths. It is commonly assumed that $b \approx a$ and $\sin \theta \approx \theta$ so that the $r(\theta \sin \theta)^{1 / 2}$ factor in the denominator of (1) is approximately $r \theta$. The computed field using this "inverse distance" factor is shown as a dashed curve. Although the form of (1) is supposedly more exact, the approximate form is quite adequate and it is impossible to discern which compares best with the experimental data.

The experimental data for a waveguide excited cylinder and the theoretical results for a dipole excited sphere shown in Fig. 2 agree very well for both surface types to $d=12 \lambda_{0}$ (or $0.6 a$ ). Sensitivity limitations prevented measurements at larger distances. The greatest difference occurs at close range $\left(d<2 \lambda_{0}\right)$. Since the residue series for $W\left(d, Z_{1}\right)$ converges slowly for small values of the 'natural' distance from the source, $x\left(=(k a ; 2)^{1 / 3} \theta\right.$ in Wait's notation), a more rapidly converging power series based upon the flat earth attenuation function should be used $[1$, p. 183], [16], [17].

Similarly, the theoretical height-gain for a dipole excited sphere and homogeneous paths was calculated using (2) at distances $d$, of 30,50 , and $70 \mathrm{~cm}$, and these are compared with experimental data in Fig. 3 . The theory agrees well with the experimental data except for small heights $\left(h_{2}<1 \mathrm{~cm}\right)$ and large heights extending far into the illuminated region. In each case the center of the penumbra region is shown as a vertical dashed line separating the shadow and the illuminated regions.

The approximation of replacing the Hankel function of order $\nu$ with the Airy function $w_{1}\left(t_{m}\right)$ in forming the residue series $[11,(69)]$ requires that $\left|t_{m}\right| \ll(2 k a)^{1 / 3}$, 


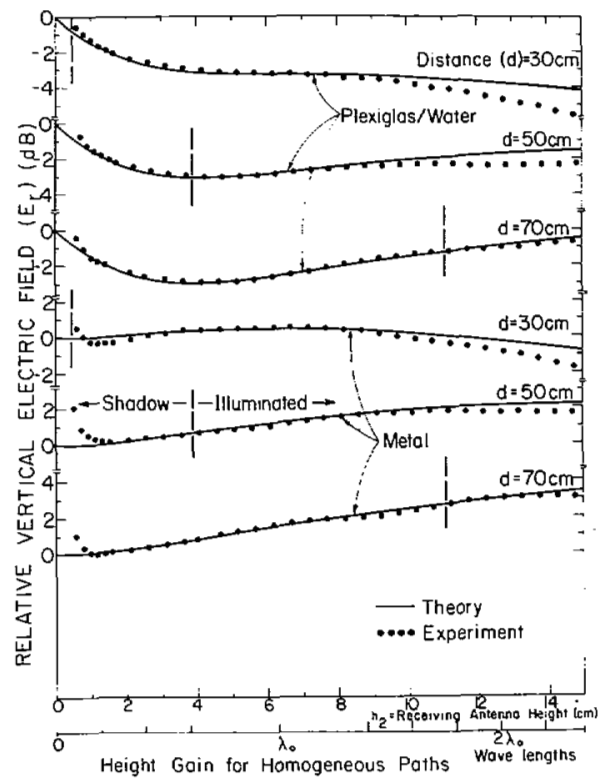

Fig. 3. Height gain data for propagation over cylindrical (experimental) and spherical (theory) homogeneous paths of either metal or Plexiglas over water. Frequency $=4.765 \mathrm{GHz}\left(\lambda_{0}=6.30 \mathrm{~cm}\right)$, $h_{1}=1.5 \mathrm{~cm}$, and $a=125 \mathrm{~cm}\left(19.85 \lambda_{0}\right)$.

where $t_{m}$ is the root of the $m$ th mode. This in turn places constraints upon $h_{1}$ and $h_{2}$, depending upon $k a$ and $\Delta_{1}$ $\left(=Z_{1} / \eta_{0}\right)$ in a rather complicated way $[1$, p. 187], [11, p. 2858]. Essentially, this means that in the shadow and penumbra regions the low-order modes of lowest attenuation adequately describe the field, but in the illuminated region the residue series converges very slowly.

In Fig. 3 it is interesting to note that the residue series is still quite reasonable even though the restriction $\left|t_{m}\right| \ll$ $(2 k a)^{1 / 3}$ is violated for $m \geqq 2$. Here, a maxinum of 50 terms was used for $d=30 \mathrm{~cm}$, and a maximum of 10 terms was used for $d=50$ and $70 \mathrm{~cm}$, corresponding to the maximum height of $h_{2}=2.4 \lambda_{0}$. Indeed, this is an unexpected result and indicates that even though the series converges slowly, it converges rather accurately for heights extending well into the illuminated region, in spite of the fact that the higher modes do not satisfy $\left|t_{m}\right| \ll(2 k a)^{1 / 3}$.

When the height of the scattering diode is less than $1 \mathrm{~cm}$, the experimental error is caused by the coupling effect between the surface and the dipole as mentioned in Section II, and is of no consequence. Except for this lnown error, the agreement in all cases is within $0.3 \mathrm{~dB}$ in the shadow and penumbra regions, of which $0.2 \mathrm{~dB}$ may be instrumentation error.

When $x$ is large compared to unity the residue series converges rapidly, the first term is then dominant and the height-gain factor for small heights $\left(y_{2}{ }^{2} \ll 1\right.$, where $y_{2}=$ $(2 / k a)^{1 / 3} k h_{2}$ in Wait's notation, [1, p. 199]) can be approximated by the simple form

$$
G\left(h_{2}\right)=1+i \hbar \Delta_{1} h_{2} .
$$

This adequately describes the height gain in Fig. 3 for all values of $d$ shown if $y_{2}{ }^{2} \ll 1$ (or equivalently, $\left(h_{2} / \lambda_{0}\right)^{2} \ll$
0.4) for both Plexiglas and water and metal media. An expression identical to (3) is also obtained for small curvature provided $k h_{2}{ }^{2} \ll 2 d[1$, p. 183], and has been experimentally confirmed for a flat earth $[6]$.

\section{B. Mixed Paths}

For $d>d_{1}$, the boundary scatters the $m$ th incident mode into an infinite number of $n$ modes characterized by a scattering coefficient $S_{n, m}$, and so the residue series solution for $W^{\prime}\left(d, Z_{1}, Z_{2}\right)$ takes the form of a double summation given by $[1,(132)],[11,(96)],[12,(35)]$. Each scattered mode contains a factor $\exp \left[-i\left(2-x_{1}\right) t_{n}{ }^{(2)}\right]$ where $t_{n}^{(2)}$ denotes the root of the $n$th mode corresponding to the second medium with surface impedance $Z_{2}$, and $x_{1}=(k a / 2)^{1 / 3} d_{1} / a$ is the 'natural' distance from the source to the boundary. Thus this series does not converge rapidly near the boundary where $\left(x-x_{1}\right)$ is small, for the same reason that the series for $W\left(d, Z_{1}\right)$ converges slowly for small $x$, i.e., near the source. With this one additional restriction, the limitations on $W^{\prime}\left(d, Z_{1}, Z_{2}\right)$ are similar to those already noted for $W\left(d, Z_{1}\right)$.

Since convergence of the residue series is poor for small $\left(x-x_{1}\right)$, one of several alternative one-dimensional convolution integral forms is more useful $[1$, p. 186], [12], $[17$, p. 403]. The $W$ functions in the integrand can be computed from the more rapidly converging power series when the natural distances $(x)$ are small compared to unity. However, it should be mentioned that these integral expressions are also invalid within about one wavelength behind the boundary owing to the dropping of the induction field in the formulation $[3],[4]$. Furthermore, the use of the surface impedance is invalid, but this error is considerably less, especially if the second medium has a large index of refraction referred to air.

The measured and calculated $E_{r}$ for propagation over two-section mixed paths consisting of Plexiglas and water and metal are shown in Figs. 4 and 5 , with $h_{2}$ as the parameter. Again, the solid and dashed curves show only a very slight difference in the way the inverse distance can be calculated.

In Fig. 4 the surface wave dominates on the first medium, being converted into a radiated wave over the second medium (metal), and vice versa in Fig. 5 . The theoretical curves agree very well with experimental data over the entire range $\left(2 \lambda_{0}<d\right)$ and all values of $h_{2}$, except for $d>11 \lambda_{0}$ in Fig. $\bar{b}$. Here the field appears to rise and fall slowly, a characteristic which is typical of the interference pattern of two forward traveling waves with slightly different phase velocities. This anomaly is not due to reflections from the sides of the model since it is approximately forty Fresnel zones wide when $d^{\prime}=11 \lambda_{0}[1, p$. $208],[12]$. This was also checked experimentally by placing microwave absorbing materials along the entire model circumference with no observable effect. Furthermore, it was not observed when the path was homogeneous (Fig. 2) or when the second medium was metal (Fig. 4), but was consistently observed for the combinations of 


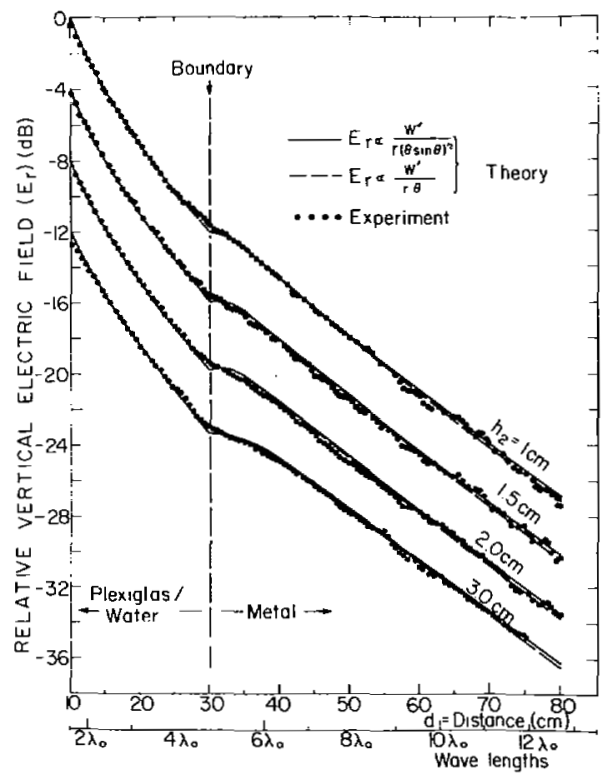

Fig. 4. Dipole excited groundwave field versus distance $d(=a \theta)$ over two-section cylindrical (experimental) and spherical (theory) mixed paths composed of Plexiglas over water and metal. Frequency $=4.765 \mathrm{GHz}\left(\lambda_{0}=6.30 \mathrm{~cm}\right)$, boundary at $d_{\mathrm{i}}=30 \mathrm{~cm}$, $h_{1}=1.5 \mathrm{~cm}$, and $a=125 \mathrm{~cm}\left(19.85 \lambda_{0}\right)$.

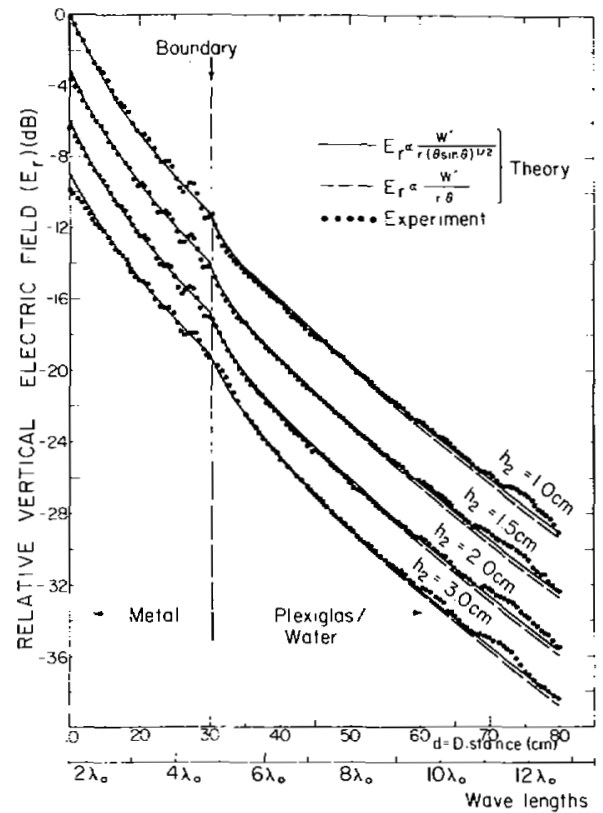

Fig. 5. Dipole excited groundwave field versus distance $d(=a \theta)$ over two-section cylindrical (experimental) and spherical (theory) mixed paths composed of metal and Plexiglas over water. Frequency $=4.765 \mathrm{GHz}\left(\lambda_{0}=6.3 \mathrm{~cm}\right)$, boundary at $d_{1}=30 \mathrm{~cm}$, $h_{1}=1.5 \mathrm{~cm}$, and $a=125 \mathrm{~cm}\left(19.85 \lambda_{0}\right)$.

media shown in lig. 5. Moving the boundary to $d_{1}=$ $40 \mathrm{~cm}$ caused the anomaly to appear at $d=9.5 \lambda_{0}$. In lig. $5, d_{1}=30 \mathrm{~cm}$ and the anomaly is first observed at $d=11 \lambda_{0}$ where the first liresnel zone has a maximum width of about $15 \mathrm{~cm}\left(2.4 \lambda_{0}\right)$. Since the incident field along this width of the boundary is not uniform, it is quite possible that there is some focusing effect which is not predicted by the dipole excited sphere theory as suggested in Section III-A. Such an effect would be most pronounced when $d$ is comparable to $a$, the boundary is about midway along the path, and the second medium is capable of supporting a surface wave well, as in the case of Plexiglas and water in Fig. 5. In any event, this experiment suggests that the accuracy suffers where the mixed path theory for a dipole excited sphere is applied to a dipole excited cylinder if $d$ is comparable to $a$, and for similar combinations of media. However, this is not a severe limitation when propagating over the real earth since the earthionosphere waveguide modes are prominent.

As noted earlier, the double series for $W^{\prime}\left(d, Z_{1}, Z_{2}\right)$ converges poorly immediately behind the boundary and it was only possible to compute the field over the second medium beyond $5 \mathrm{~cm}$ from the boundary $(d>35 \mathrm{~cm})$. The equivalent integral [1, p. 186], [12], [17, p. 403] form was, therefore, used for $d_{1}<d<d_{1}+\lambda_{0}$, where it can be seen that the agreement between experiment and theory is good even though the applicability of the integral form there is questionable from a theoretical standpoint. Note that the familiar recovery effect immediately to the right of the boundary in ligs. 4 and 5 spreads over a larger region as $h_{2}$ increases.

Waves reflected to the left of the boundary are quite obvious in Fig. 5, while they are almost unobservable in Y'ig. 4. Almost identical experimental results were obtained previously when propagating over a flat surface comprised of the same mixed paths [7]. Although Wait [11] has given formulas for the field reflected from an abrupt boundary on a cylindrical surface, we were not successful in making calculations since these reflections are only significant near the boundary where the residue series does not converge for the same reasons mentioned above. However, we did compare the reflected field with Wait's [14] formula for propagation over a smooth abrupt boundary separating two media, both having a small normalized surface impedance. But this solution only predicts a change in the reflected wave sign, the magnitude of the reflection being the same for an interchange of media. Clearly, this is not the case for the experimental data shown in ligs. 4 and 5 . The actual reflected wave is somewhat larger than the wave predicted using Wait's formula for the case shown in lig. 5 , and smaller in Fig. 4.

The cause of this disparity is uncertain, but the abrupt boundary used here did have a discontinuity in height equal to the thickness of the adhesive backed aluminum foil $(0.0125 \mathrm{~cm})$. This height difference was not present in some previous experimental studies [3], [4] where the comparison of experimental data with the computed reflected ficld revealed no apparent disparity. Nevertheless, we do feel the measurement techniques used here are more reliable, and so additional experiments are being designed to answer this question in a future note.

The theoretical height gain $G\left(h_{2}\right)$ over the second medium was calculated using the residue series for $W^{\prime}\left(d, Z_{1}, Z_{2}\right)$ in (2) for boundaries located at 30 and $40 \mathrm{~cm}$ from the source and observation distances of 50 and $70 \mathrm{~cm}$, respectively, as shown in Fig. 6. The conclusions are the same as those drawn from the homogeneous 


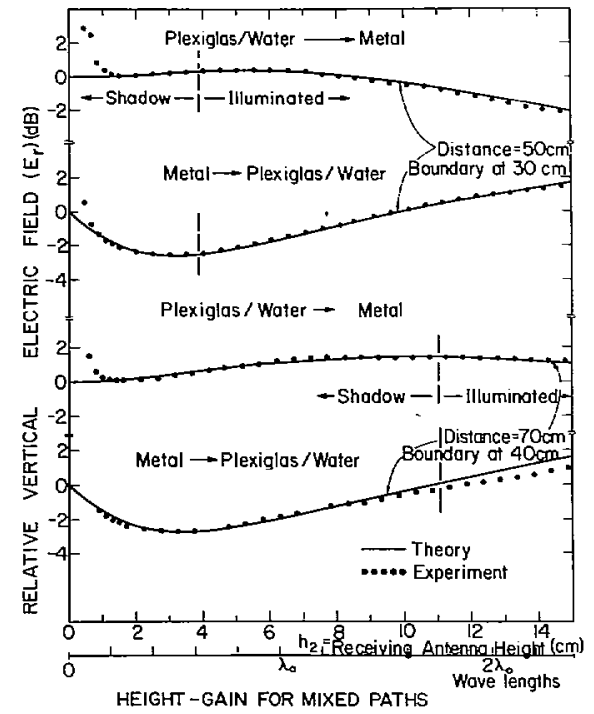

Fig. 6. Height gain for propagation over two-section cylindrical (experimental) and spherical (theory) mixed paths composed of metal and Plexiglas over water and vice versa. Frequency = $4.765 \mathrm{GHz}\left(\lambda_{0}=6.3 \mathrm{~cm}\right)$, boundary at 30 or $40 \mathrm{~cm}, h_{1}=1.5 \mathrm{~cm}$, and $a=125 \mathrm{~cm}\left(19.85 \lambda_{0}\right)$.

case, Fig. 3, i.e., the theoretical curves agree with experimental data extending well into the illuminated region where a large number of terms were needed and $\left|t_{n}{ }^{(2)}\right| \ll$ $(2 k a)^{1 / 3}$ was not satisfied for $n>2$. The approximate form (3) holds for $y_{2}^{2} \ll 1$ (i.e., $\left(h_{2} / \lambda_{0}\right)^{2} \ll 0.4$ ).

Note that the height gain over the second medium in Fig. 6 has the same characteristics as the corresponding homogeneous path (Fig. 3 ) within the shadow region, and in the penumbra and illuminated regions both media effect the height gain. Therefore, the field strength could be predicted graphically for small heights by adjusting the field strength on the surface with the height gain factor over the corresponding homogeneous path. Similar conclusions have been made for groundwave propagation over mixed paths on a flat surface [7], but in the present case the range of heights is apparently much greater. For the flat earth, this procedure for estimating the height gain over the second medium is only valid at heights where a significant surface wave exists.

\section{CONCLUSIONS}

These experimental results suggest that the residue series solution for a dipole excited sphere accurately describes propagation over a dipole excited cylinder for homogeneous paths and distances extending to at least 0.6a. For mixed paths the equivalence of the two problems appears questionable when $d>0.5 a$ and the second medium supports a surface wave well.

Aside from this, the residue series formulas for groundwave propagation over a spherical surface have been shown to be highly accurate in the shadow and penumbra regions (error $<0.3 \mathrm{~dB}$ for these models), and reasonably accurate for the low part of the illuminated region for both homogeneous and two-section mixed paths (Figs. 3 and 6 , respectively). In the illuminated region or very near the source or a boundary, an excessive number of residue terms is needed. Immediately behind the boundary the equivalent integral solution is useful.

When a wave is diffracted around a curved surface and crosses a boundary, it rapidly acquires the characteristics of the second medium, enabling the magnitude of the height gain to be estimated as if the second medium constituted the entire path.

These model experiments have shown that the theory is accurate for $a / \lambda_{0}$ as small as 20 and for mixed paths with a large contrast in surface impedance. It should be even more accurate for larger scale situations such as VLF propagation over the earth where $a / \lambda_{0}$ is considerably larger and typical surface impedance contrasts between mixed paths are less, assuming, of course, that path segments are homogeneous, isotropic and smooth.

\section{ACKNOWLEDGMENT}

The helpful comments by Dr. J. R. Wait and the typing assistance of $J$. Lokken are also appreciated.

\section{REFERENCES}

[1] J. R. Wait, "Electromagnetic surface waves," in Advances in Radio Research, J. A. Saxton, Ed. London, England: Academic Press, 1964, pp. 175-209, vol. 1.

[2] J. R. Wait and A. M. Conda, "Pattern of an antenna on a curved lossy surface," IRE Trans. Antennas Propagat., vol. AP-6, pp. 348-359, Oct. 1958.

[3] R. J. King and S. W. Maley, "Model experiments on propagation of groundwaves across an abrupt boundary at perpendicular incidence," Radio Sci., vol. 69D, pp. 1375-1381, Oct. 1965.

[4] R. J. King, S. W. Maley, and J. R. Wait, "Experimental and theoretical studies of propagation of groundwaves across mixed paths," in Electromagnetic Theory, J. Brown, Ed. New York: Pergamon Press, 1967.

[5] R. J. King and J. Radtke, "Measurement of surface impedance at microwave frequencies," Electron. Lett., vol. 4, no. 14, pp. 296-298, July 12, 1968.

[6] R. J. King, S. H. Cho, D. L. Jaggard, and V. Sokolov, "Height gain experimental data for groundwave propagation; Part 1homogeneous paths," Radio Sci., vol. 8, no. 1, pp. 7-15, Jan. 1973.

[7] - , "Height gain experimental data for groundwave propagation; Part 2-heterogeneous paths," Radio Sci., vol. 8, no. 1, pp. 17-22, Jan. 1973.

[8] R. J. King and G. A. Schlak, "Groundwave attenuation function for propagation over a highly inductive earth," Radio Sci., vol. 2, no. 7, pp. 687-693, July 1967.

[9] J. R. Wait, "The propagation of electromagnetic waves along the earth's surface," in Electramagnetic Waves, R. E. Langer, Ed. Madison, Wis.: Univ. Wisconsin Press, pp. 258-267 and $277-290,1962$.

[10] N. A. Logan and K. S. Yee, "A mathematical model for diffraction by convex surfaces," in Electromagnetic Waves, R. E. Langer, Ed. Madison, Wis.: Univ. Wisconsin Press, pp. 139 $180,1962$.

[11] J. R. Wait, "Propagation of electromagnetic waves over a smooth multisection curved earth-an exact theory," $J$. Math. Phys., vol. 11, no. 9, pp. 2851-2860, Sept. 1970.

[12] -, "Recent analytical investigations of electromagnetic ground wave propagation over inhomogeneous earth models," in Proc. $A G A R D$, Mar. 1974.

[13] - , private correspondence, Nov. 16, 1973.

[14] ' "Oblique propagation of groundwaves across a coastline, Part'I,"J. Res. Nat. Bur. Stand., vol. 67D, no. 6, pp. 617-624, Nov.-Dec. 1963.

[15] —-, "Nature of the electromagnetic field reflected from a coastline," Electron. Lett., vol. 1, no. 3, pp. 65-66, May 1965.

[16] J. R. Wait and K. P. Spies, "On the calculation of the groundwave attenuation factor at low frequencies," IEEE Trans. Aniennas Propagat., vol. AP-14, pp. 515-517, July 1966.

[17] K. P. Spies and J. R. Wait, "On the calculation of antenna patterns for an inhomogeneous earth," Radio $S c i$, vol. 2, no. 11, pp. 1361-1378, Nov. 1967. 